TITLE:

\title{
Dynamic van der waals theory of two-phase fluids in heat flow
}

$\operatorname{AUTHOR}(\mathrm{S}):$

Onuki, A

\section{CITATION:}

Onuki, A. Dynamic van der waals theory of two-phase fluids in heat flow. PHYSICAL REVIEW LETTERS 2005, 94(5): 054501.

ISSUE DATE:

2005-02-11

URL:

http://hdl.handle.net/2433/49918

RIGHT:

Copyright 2005 American Physical Society 


\title{
Dynamic van der Waals Theory of Two-Phase Fluids in Heat Flow
}

\begin{abstract}
Akira Onuki
Department of Physics, Kyoto University, Kyoto 606-8502, Japan

(Received 6 June 2004; revised manuscript received 5 October 2004; published 7 February 2005)

We present a dynamic van der Waals theory. It is useful to study phase separation when the temperature varies in space. We show that, if heat flow is applied to liquid suspending a gas droplet at zero gravity, a convective flow occurs such that the temperature gradient within the droplet nearly vanishes. As the heat flux is increased, the droplet becomes attached to the heated wall that is wetted by liquid in equilibrium. In one case corresponding to partial wetting by gas, an apparent contact angle can be defined. In the other case with larger heat flux, the droplet completely wets the heated wall expelling liquid.
\end{abstract}

DOI: 10.1103/PhysRevLett.94.054501

In the usual theories of phase transitions, the fluctuations of the temperature $T$ are assumed to be small and are neglected. However, there can be situations in which phase transitions occur in inhomogeneous $T$. For example, wetting properties near the gas-liquid critical point are very sensitive to applied heat flux [1,2], and boiling processes remain largely unexplored $[3,4]$. To treat such problems we propose to start with coarse-grained entropy and energy, from which the temperature is defined as a functional of the order parameter and the energy density.

Let us consider one-component fluids, where the order parameter is the number density $n$. In the van der Waals theory the energy density $e$ and the entropy $s$ per particle are given by $e=d n k_{B} T / 2-\epsilon v_{0} n^{2}$ and $s / k_{\mathrm{B}}=(d / 2) \times$ $\ln T+\ln \left(1 / v_{0} n-1\right)+$ const, respectively, in terms of $T$ and $n$ [5]. Here $v_{0}$ and $\epsilon$ represent the molecular volume and the magnitude of the attractive potential, respectively, and $d$ is the space dimensionality. To describe situations in the presence of interfaces, we generalize this description by including gradient contributions to the total entropy and the total internal energy as

$$
\begin{aligned}
& S=\int d \boldsymbol{r}\left[n s-\frac{1}{2} C|\nabla n|^{2}\right], \\
& \mathcal{E}=\int d \boldsymbol{r}\left[e+\frac{1}{2} K|\nabla n|^{2}\right] .
\end{aligned}
$$

The gradient terms represent a decrease of the entropy and an increase of the energy due to inhomogeneity of $n$. Here the internal energy density is written as

$$
\hat{e}=e+K|\nabla n|^{2} / 2
$$

including the gradient part. We assume that the coefficients $C$ and $K$ are simply constants and that $s$ in Eq. (1) depends on $n$ and $e$ as

$$
s=k_{\mathrm{B}} \ln \left[\left(e / n+\epsilon v_{0} n\right)^{d / 2}\left(1 / v_{0} n-1\right)\right]+\text { const. }
$$

This relation follows from the van der Waals theory. Our scheme starts with Eqs. (1), (2), and (4), where the tem-

PACS numbers: 47.20.Dr, 05.70.Ln, 44.35.+c, 64.70.Fx

perature is not yet introduced. We then define the local temperature $T=T(n, e)$ by

$$
\frac{1}{T}=\left(\frac{\delta}{\delta e} S\right)_{n}=n\left(\frac{\partial s}{\partial e}\right)_{n}
$$

where $n$ is fixed in the derivatives. This definition of $T$ is analogous to that in a microcanonical ensemble. We also define the local chemical potential $\hat{\mu}$ (per particle) including the gradient contributions by

$$
\hat{\mu}=-T\left(\frac{\delta S}{\delta n}\right)_{\hat{e}}=\mu-T \nabla \cdot \frac{M}{T} \nabla n,
$$

where $\hat{e}$ in Eq. (3) is fixed, $\mu=-T(\partial(n s) / \partial n)_{e}$, and

$$
M=C T+K .
$$

Maximization of $S$ under a fixed total particle number $\int d \boldsymbol{r} n$ and a fixed total energy $\mathcal{E}$ leads to the equilibrium conditions $T=$ const and $\hat{\mu}=$ const. As first derived by van der Waals $[5,6]$, the equilibrium interface density profile $n=n(x)$ is determined by $\mu(n, T)-M d^{2} n / d x^{2}=$ $\mu_{\mathrm{cx}}$ with the surface tension $\gamma=M \int_{-\infty}^{\infty} d x(d n / d x)^{2}$, where $\mu_{\mathrm{cx}}$ is the chemical potential on the coexistence curve [7].

Next we set up the dynamic equations [8]. Hereafter gravity is neglected. The mass density $\rho=m n$ ( $m$ being the particle mass) obeys $\partial \rho / \partial t=-\nabla \cdot(\rho \boldsymbol{v})$, where $\boldsymbol{v}$ is the velocity field. The momentum density $\rho \boldsymbol{v}$ obeys

$$
\frac{\partial}{\partial t} \rho \boldsymbol{v}=-\nabla \cdot(\rho \boldsymbol{v} \boldsymbol{v})-\nabla \cdot(\overleftrightarrow{\Pi}-\overleftrightarrow{\sigma})
$$

The $\overleftrightarrow{\Pi}=\left\{\Pi_{i j}\right\}$ is the reversible stress tensor invariant with respect to time reversal $\boldsymbol{v} \rightarrow-\boldsymbol{v}$. The $\overleftrightarrow{\sigma}=\left\{\sigma_{i j}\right\}$ is the dissipative stress tensor of the form $\sigma_{i j}=\eta\left(\nabla_{i} v_{j}+\nabla_{j} v_{i}\right)+$ $(\zeta-2 \eta / d) \delta_{i j} \nabla \cdot \boldsymbol{v}$ with $\nabla_{i}=\partial / \partial x_{i}$ in terms of the viscosities $\eta$ and $\zeta$. The (total) energy density $e_{\mathrm{T}}=\hat{e}+$ $\rho \boldsymbol{v}^{2} / 2$ including the kinetic part is governed by

$$
\frac{\partial}{\partial t} e_{\mathrm{T}}=-\nabla \cdot\left[e_{\mathrm{T}} \boldsymbol{v}+(\overleftrightarrow{\Pi}-\overleftrightarrow{\sigma}) \cdot \boldsymbol{v}\right]+\nabla \cdot(\lambda \nabla T),
$$


where $\lambda$ is the thermal conductivity. We construct the stress tensor $\Pi_{i j}$ such that the entropy production rate within the fluid assumes the usual form [8],

$$
\frac{\partial}{\partial t} S=\int d \boldsymbol{r} \frac{1}{T}\left[\nabla \cdot \lambda \nabla T+\sum_{i j} \sigma_{i j} \nabla_{i} v_{j}\right] .
$$

If there is no heat flow from outside, the above quantity becomes non-negative-definite. To obtain Eq. (10) we need to require the general relation,

$$
\nabla \cdot\left(\frac{1}{T} \overleftrightarrow{\Pi}\right)=-n \nabla\left(\frac{\delta}{\delta n} S\right)_{\hat{e}}-\hat{e} \nabla\left(\frac{\delta}{\delta \hat{e}} S\right)_{n} .
$$

Using the van der Waals pressure $p=n k_{B} T /\left(1-v_{0} n\right)-$ $\epsilon v_{0} n^{2}$ we then find

$$
\Pi_{i j}=\left(p+p_{1}\right) \delta_{i j}-M \nabla_{i} n \nabla_{j} n,
$$

where $p_{1}=M\left(n \nabla^{2} n+|\nabla n|^{2} / 2\right)+T n \nabla n \cdot \nabla(M / T)$.

As an example, we consider a bubble with radius $R$ in heat flow. Thermocapillary bubble migration has long been observed, especially in space [9-11]. Theoretically, however, the bubble motion has been examined in the absence of first-order phase transition at the interface such as in the case of air bubbles in silicone oil [9]. In such cases the temperature dependence of the surface tension $\gamma$ causes a bubble velocity of order $v_{\mathrm{Y}}=R \gamma_{1}(d T / d z)_{\infty} / \eta$ in a given temperature gradient $(d T / d z)_{\infty}$ (Marangoni effect), where $\gamma_{1}=-d \gamma / d T>0$ and $\eta$ is the viscosity in liquid. In one-component fluids the effect is drastically altered due to latent heat generation or absorption at the interface [12]. We point out that a convective velocity of order $v_{\mathrm{O}}=$ $\lambda(d T / d z)_{\infty} / \rho^{\prime} T \Delta s$ inside the bubble is sufficient to carry the applied heat flux, where $\rho^{\prime}$ is the mass density in gas, $\Delta s$ is the entropy difference between gas and liquid per unit mass, and $\lambda$ is the thermal conductivity in liquid. Note that the ratio

$$
M_{1}=v_{\mathrm{Y}} / v_{\mathrm{O}}=R \gamma_{1} \rho^{\prime} T \Delta s / \eta \lambda
$$

is a very large number of order $R / a$ with $a$ being a microscopic length. With phase change the bubble velocity is of order $v_{\mathrm{O}}$ and is very slow [12]. Furthermore, the temperature inside the bubble should be nearly homogeneous due to the latent heat convection. This is a natural consequence in one-component fluids without contamination [1,12], because the pressure should become nearly homogeneous outside the bubble and the fluid near the interface should be close to the coexistence curve in the $p-T$ phase diagram.

In our simulation we set $K=0$, and then the interface thickness is $\ell=\left(C v_{0} / 2 k_{\mathrm{B}}\right)^{1 / 2}$ far from the critical point [since $\left(\mu-\mu_{\mathrm{cx}}\right) / k_{\mathrm{B}} T$ is of order 1 for $n \sim v_{0}^{-1}$ ]. Space is measured in units of $\ell$, and the system length is $L=202 \ell$. Time is measured in units of $t_{0}=m \ell^{2} / \eta v_{0}$. Here we assume constant viscosities with $\eta=\zeta$. The strength of dissipation is represented by the normalized viscosity $\eta^{*}=v_{0} \eta / \ell \sqrt{m \epsilon}$. We set $\eta^{*}=0.1^{1 / 2}$. On all the boundary walls we assume $[13,14]$

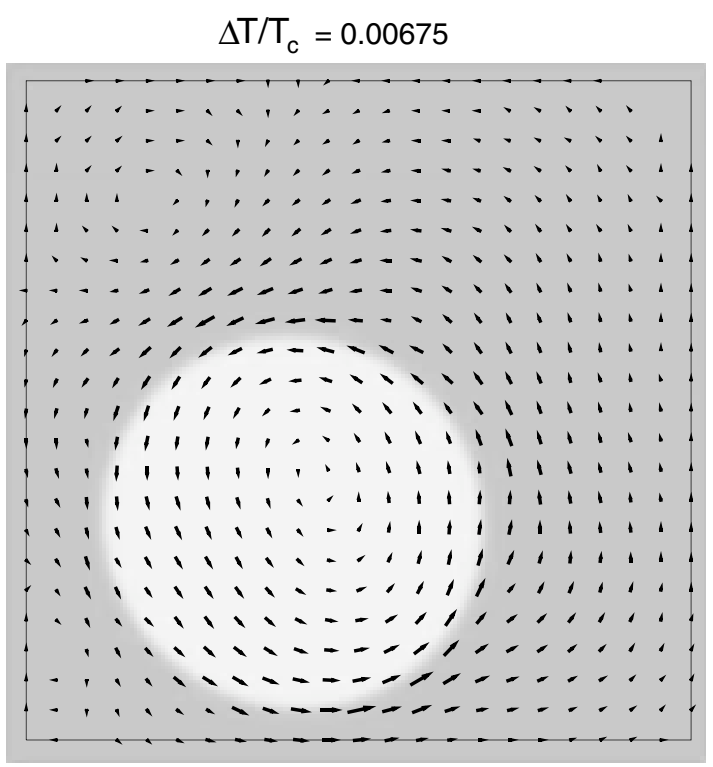

FIG. 1. Suspended gas droplet in a steady state in heat flow with $\Delta T=0.00675 T_{\mathrm{c}}$. The arrows indicate the velocity.

$$
n=n_{\mathrm{w}}+\ell_{\mathrm{w}} \boldsymbol{\nu} \cdot \nabla n,
$$

where $\boldsymbol{\nu}$ is the inward normal unit vector at the surface. We set $n_{\mathrm{w}}=5 n_{\mathrm{c}} / 2$ and $\ell_{\mathrm{w}}=2 \sqrt{2} \ell$, for which the wall is wetted by liquid in equilibrium in our simulation. We prepared an initial equilibrium state with a circular gas bubble with radius 50 placed at the center of the cell. The temperature was at $T=0.875 T_{\mathrm{c}}$, where the density is $0.37 n_{\mathrm{c}}$ in gas and $1.74 n_{\mathrm{c}}$ in liquid. The bottom boundary $(y=0)$ was then increased by a constant $\Delta T$, while the top boundary $(y=L)$ was held at the initial temperature $T_{\mathrm{t}}=$ $0.875 T_{\text {c }}$. (Here we use "bottom" and "top" though no gravity is assumed.) The side boundaries ( $x=0$ and $L)$ are insulating $(\boldsymbol{\nu} \cdot \nabla T=0)$. Furthermore, the density dependence of the thermal conductivity is crucial away from criticality [15], so we set $\lambda=\left(v_{0} \eta / k_{\mathrm{B}} m\right) n$ with the ther-

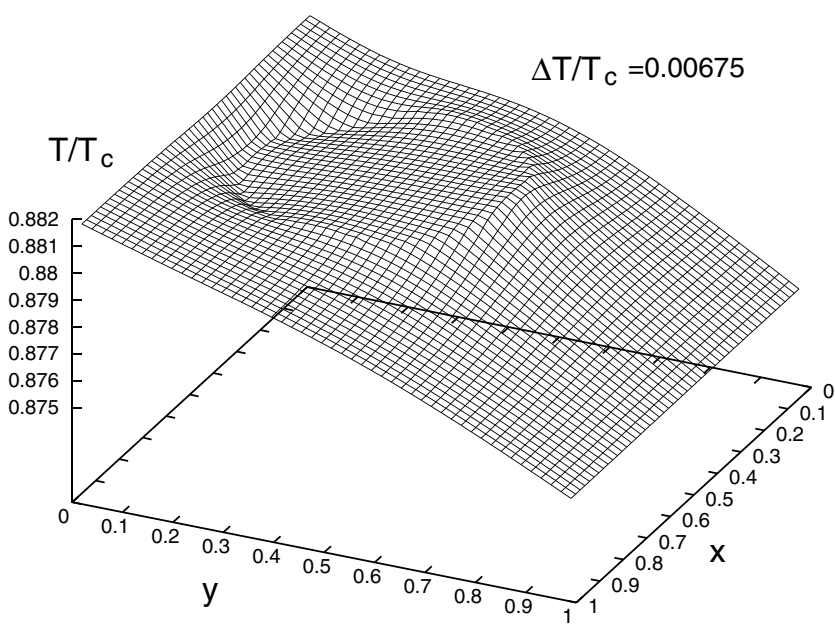

FIG. 2. The temperature in the steady state in Fig. 1. It is flat within the gas droplet due to latent heat transport. 


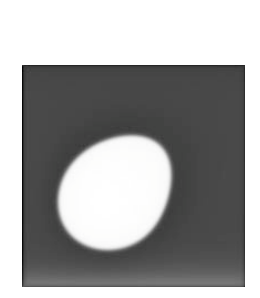

$t=1000$

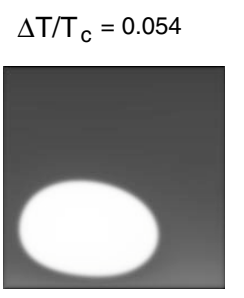

$t=7000$

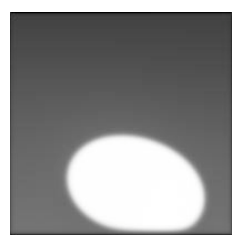

$\mathrm{t}=80000$
FIG. 3. Migration of a gas droplet for $\Delta T=0.054 T_{\mathrm{c}}$. It apparently wets the bottom partially in the steady state (left), where a thin liquid layer is still sandwiched.

mal diffusivity $D_{T}$ being of order 1 (in units of $\ell^{2} / t_{0}=$ $\left.v_{0} \eta / m\right)$. Then $\lambda$ in liquid is larger than that in gas by 5 times.

(i) First, for very small $\Delta T$, the bubble position is shifted toward the bottom without shape changes. Figure 1 shows such a steady state at $\Delta T=0.00675 T_{\mathrm{c}}$ taken at $t=3.4 \times$ $10^{4}$ after the application of heat flux. Here the migration is stopped by the repulsion from the walls due to the wetting condition in Eq. (14). The velocity field $\boldsymbol{v}$ is almost parallel to the interface, but there is a small velocity component through the interface. Thus, at the interface, first-order phase transition takes place, and latent heat is generated or absorbed. As demonstrated in Fig. 2, latent heat transport is so efficient that the temperature gradient almost vanishes within the bubble.

(ii) Second, for the case $\Delta T=0.054 T_{\mathrm{c}}$, Fig. 3 shows droplet migration toward the bottom. Figure 4 displays the velocity field in the steady state taken at $t=8 \times 10^{4}$. The velocity component through the interface is more increased than in Fig. 1, again resulting in a flat temperature inside the droplet as in Fig. 5. As can be seen in Figs. 4 and 5, a thin liquid layer is sandwiched between the bottom bound-

$\Delta \mathrm{T} / \mathrm{T}_{\mathrm{c}}=0.054$

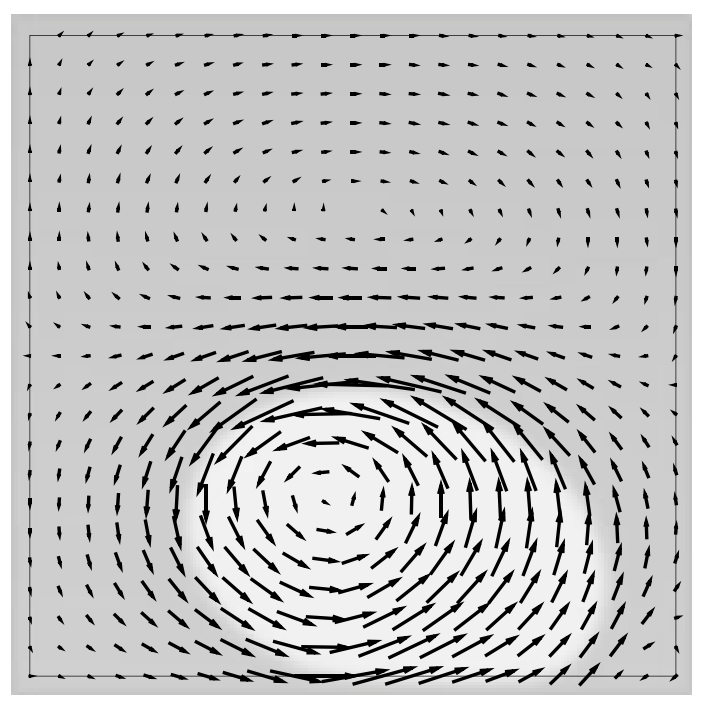

FIG. 4. The velocity field around the gas droplet at $t=8 \times$ $10^{4}$ in Fig. 3.

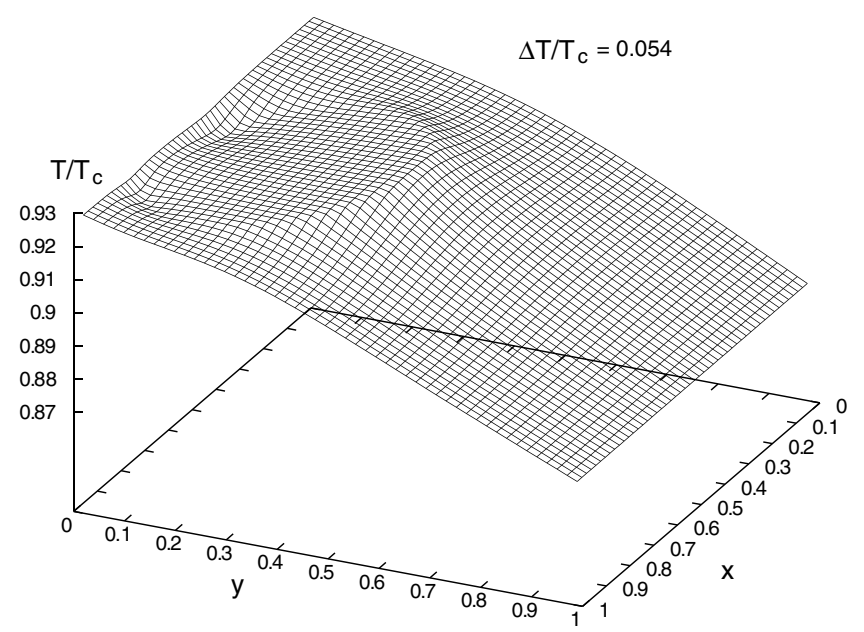

FIG. 5. The temperature in the nearly steady state in Fig. 4.

ary and the droplet. The thickness of this layer is so thin that we can define an apparent contact angle $\theta_{\text {eff }}$, which is a decreasing function of $\Delta T$. In accord with these results, Garrabos et al. observed that gas spreads on a heated wall initially wetted by liquid and exhibits an apparent contact angle [1].

(iii) Third, we increased $\Delta T$ to $0.10125 T_{\mathrm{c}}$, where the bottom temperature is $0.97 T_{\mathrm{c}}$. As shown in Fig. 6, phase separation occurred to produce a gas domain at the bottom, into which the suspended droplet was finally absorbed. In the steady state in Fig. 7, the bottom is covered by a gas layer or is completely wetted by gas [16], but the interface with the bulk liquid is still curved and a small velocity field is induced. As can be seen in Fig. 8 taken at $t=3 \times 10^{4}$, the temperature gradient within the gas layer becomes steeper than that in the liquid. It is well known that a gas film on a heated wall suppresses heat transfer to the bulk liquid and, as it thickens, film boiling is induced in gravity [3].

In the steady states the velocity is of order $v_{\mathrm{O}} \sim$ $D_{T} \Delta T / T L$ in terms of $D_{T}=\lambda / \rho C_{p}$ from $C_{p} \sim \Delta s$. As a result, the Reynolds number $\left(\sim \operatorname{Pr}^{-1} R \Delta T / L T\right)$ is very small, where the Prandtl number $\operatorname{Pr}=v_{0} \eta / m D_{T}$ is here taken to be of order 1. In fact, in Figs. 1, 4, and 7, the variance $\sqrt{\left\langle\boldsymbol{v}^{2}\right\rangle}$ is $0.12,1.9$, and 0.63 , respectively, in units

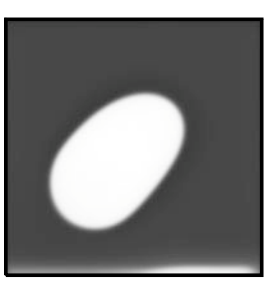

$t=300$

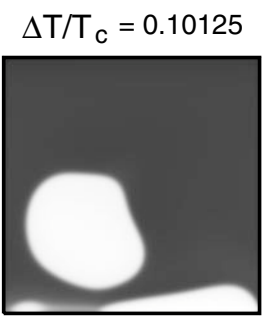

$t=800$

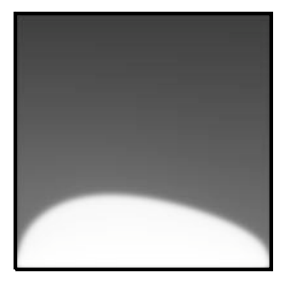

$t=30000$
FIG. 6. Time evolution for $\Delta T=0.10125 T_{\mathrm{c}}$. Phase separation occurs at the bottom. The bottom is completely wetted by gas in the steady state. 


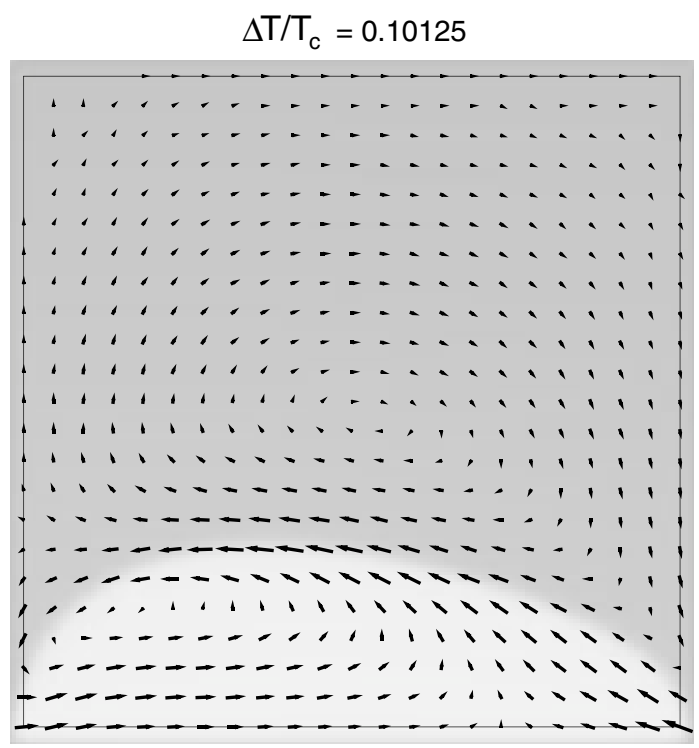

FIG. 7. The velocity field around the gas region at $t=3 \times 10^{4}$ in Fig. 6.

of $10^{-3} \ell / t_{0}$, where $\ell / t_{0}=D_{T} / \operatorname{Pr} \ell$. In our simulation the viscous dissipation is very strong even in transient states, while in the theory in Ref. [11] the inertia effect (vapor recoil) is important in bubble motion on a heated wall. This difference stems from the fact that our droplet size is very small if $\ell$ is microscopic.

In the diffuse-interface methods for crystal growth [17] and thermocapillary flow $[18,19]$, the interface width $\ell$ may be conveniently treated as a free parameter. This is because the phase field equations yield the movinginterface problems with appropriate interface boundary conditions as long as $\ell \ll R$ (= domain size). Also in fluids we may take $\ell$ as a free parameter in the range $\ell \ll R$ as far as we treat the steady state profiles (where the Marangoni effect is suppressed and the Stokes approximation is valid). However, to describe rapid fluid motions, the normalized viscosity $\eta^{*}(\propto \eta / \ell)$ should be made very small if $\ell$ is enlarged.

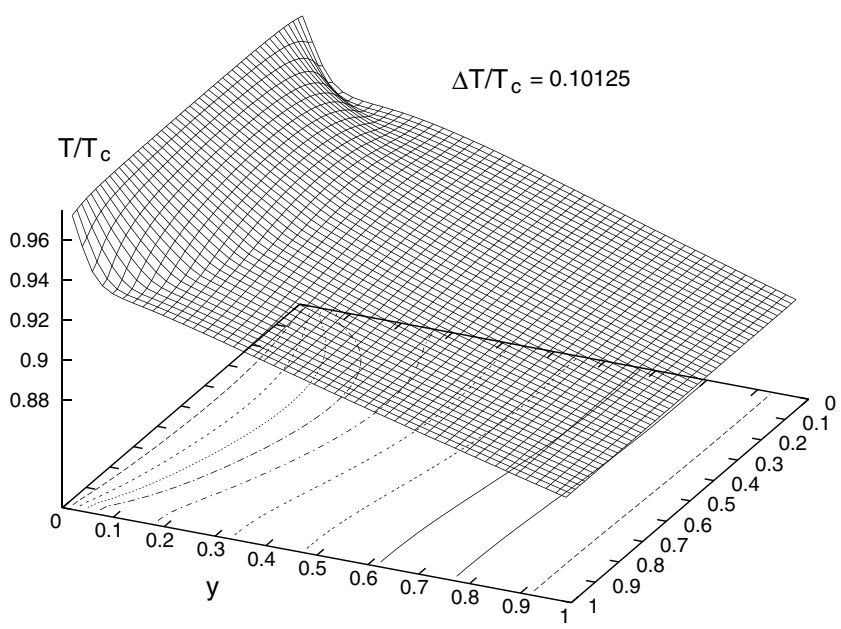

FIG. 8. The temperature in the nearly steady state in Fig. 7.
In summary, we have developed a scheme to study phase transition dynamics with inhomogeneous temperature under mass and heat fluxes. It can serve as a phase field model in fluids, though we should examine its sharpinterface limit [19]. For one-component fluids we mention wetting dynamics with evaporation and condensation $[2,13]$, as exemplified here, and boiling or convection in gravity $[4,5]$. These effects can in principle be studied in the scheme of thermocapillary hydrodynamics supplemented with interface conditions. However, hydrodynamic calculations accounting for latent heat transport have rarely been performed.

This work is supported by Grant for the 21st Century COE project (Center for Diversity and Universality in Physics) from the Ministry of Education, Culture, Sports, Science and Technology of Japan.

[1] Y. Garrabos, C. Lecoutre-Chabot, J. Hegseth, V.S. Nikolayev, D. Beysens, and J.-P. Delville, Phys. Rev. E 64, 051602 (2001).

[2] L. M. Pismen and Y. Pomeau, Phys. Rev. E 62, 2480 (2000).

[3] V.S. Nikolayev and D. A. Beysens, Europhys. Lett. 47, 345 (1999).

[4] A. Onuki, Physica (Amsterdam) 314A, 419 (2002).

[5] A. Onuki, Phase Transition Dynamics (Cambridge University Press, Cambridge, 2002).

[6] J. S. Rowlinson, J. Stat. Phys. 20, 197 (1979).

[7] For the van der Waals model $\gamma \propto M^{1 / 2}\left(1-T / T_{\mathrm{c}}\right)^{3 / 2}$ holds well for $T>0.8 T_{\mathrm{c}}$ within a few percent.

[8] L.D. Landau and E. M. Lifshitz, Fluid Mechanics (Pergamon, New York, 1959).

[9] N. O. Young, J. S. Goldstein, and M. J. Block, J. Fluid Mech. 6, 350 (1959).

[10] B. Braun, Ch. Ikier, H. Klein, and D. Woermann, Chem. Phys. Lett. 233, 565 (1995).

[11] D. Beysens, Y. Garrabos, V. S. Nikolayev, C. LecoutreChabot, J.-P. Delville, and J. Hegseth, Europhys. Lett. 59, 245 (2002).

[12] A. Onuki (to be published). The temperature gradient inside a droplet is smaller than that far from it by $1 / M_{1} M_{2}$, where $M_{2}=R \rho^{\prime} \Delta s / \gamma_{1}$ is also of order $R / a \gg 1$.

[13] P. G. de Gennes, Rev. Mod. Phys. 57, 827 (1985).

[14] S. Puri and K. Binder, Z. Phys. B 86, 263 (1992).

[15] R. B. Bird, W. E. Stewart, and E. N. Lightfoot, Transport Phenomena (Wiley, New York, 2002), p. 272.

[16] At large heat flux the density steeply changes within a few lattice points at the heated wall. Here the boundary condition Eq. (14) is questionable.

[17] G. Caginalp, Phys. Rev. A 39, 5887 (1989); R. Kobayashi, Physica (Amsterdam) 63D, 410 (1993); A. Karma and W. J. Rappel, Phys. Rev. E 57, 4323 (1998).

[18] D. Jasnow and J. Viñals, Phys. Fluids 8, 660 (1996); R. Borcia and M. Bestehorn, Phys. Rev. E 67, 066307 (2003).

[19] D. M. Anderson, G. B. McFadden, and A. A. Wheeler, Annu. Rev. Fluid Mech. 30, 139 (1998). Here an attempt of taking the sharp-interface limit for fluids is given. 